\title{
Perioperative oral supplementation with fish oil promotes liver regeneration following partial hepatectomy in mice via AMPK activation
}

\author{
HUI YAO ${ }^{1}$, XIAO FU ${ }^{1}$, XUEJIAN ZI ${ }^{2}$, WENJUN JIA ${ }^{1}$ and YUDONG QIU ${ }^{1}$ \\ ${ }^{1}$ Department of Hepatopancreatobiliary Surgery, Nanjing Drum Tower Hospital, Medical School of Nanjing University, \\ Nanjing, Jiangsu 210008; ${ }^{2}$ Department of Hepatopancreatobiliary Surgery, Nanjing Drum Tower Hospital, \\ Clinical College of Nanjing Medical University, Nanjing, Jiangsu 210029, P.R. China
}

Received January 13, 2016; Accepted September 20, 2017

DOI: $10.3892 / \mathrm{mmr} .2017 .8362$

\begin{abstract}
The present study aimed to observe the effects of perioperative oral supplementation with fish oil (FO) on liver regeneration in mice and examine the potential mechanism. A total of 120 male ICR mice were randomly divided into 5 groups: Sham, Control, fish oil (FO), Compound C [the AMP-activated protein kinase (AMPK) inhibitor dorsomorphin], and Compound $\mathrm{C}+\mathrm{FO}$. Changes in liver function, alterations in hepatocyte proliferation and in the expression of polarization markers, and activation of AMPK signaling were examined following partial hepatectomy $(\mathrm{PH})$. The results demonstrated that restoration of serum alanine aminotransferase (ALT) and total bilirubin (TBIL) levels were significantly faster in FO-treated mice compared with Control mice, and this effect was suppressed by treatment with Compound C. FO-treated mice exhibited increased numbers of Ki-67 positive hepatocytes and their postoperative liver-to-body weight ratio was significantly increased compared with the Control mice, which was also suppressed by co-treatment with the AMPK inhibitor. Furthermore, protein expression of Occludin, Claudin-3, tight junction protein 1 and bile salt export pump was gradually increased in FO-treated mice compared with Control, whereas Compound $\mathrm{C}$ treatment reversed this effect. Therefore, the present study revealed that perioperative oral supplementation
\end{abstract}

Correspondence to: Professor Yudong Qiu, Department of Hepatopancreatobiliary Surgery, Nanjing Drum Tower Hospital, Medical School of Nanjing University, 321 Zhongshan Road, Nanjing, Jiangsu 210008, P.R. China

E-mail: yudongqiu510@163.com

Abbreviations: FO, fish oil; n-3 PUFA, omega-3 polyunsaturated fatty acid; BSEP, bile salt export pump; AMPK, AMP-activated protein kinase; PH, partial hepatectomy; ALT, alanine aminotransferase; AST, aspartate aminotransferase; TBIL, total bilirubin; ALB, albumin; CRP, C-reactive protein

Key words: fish oil, partial hepatectomy, AMP-activated protein kinase, liver regeneration with FO may promote liver regeneration and improved liver function in mice following PH through AMPK activation.

\section{Introduction}

The human liver exerts vital functions, including absorbing metabolites from the intestine, regulating glucose and lipid metabolism and biotransforming xenobiotics. The liver is one of the few organs that can regenerate itself following partial ablation or liver damage. Although hepatocytes have extensive regenerative capacity, there are many diseases in which this capacity is insufficient to compensate for the loss of hepatocytes and liver function (1-3). Over the past three decades, liver regeneration has been studied extensively and several regulatory pathways have been identified, which provided regenerative alternatives to the liver and ensured the return of its mass and function to the size needed for the body (4-7). Previous studies involving humans and rodents have demonstrated that fish oil (FO), which is rich in omega-3 polyunsaturated fatty acids (n-3 PUFAs), ameliorates the degree of liver injury and improves liver function $(8,9)$. However, the mechanism by which dietary supplementation of FO improves liver function remains elusive.

Liver regeneration involves increased cell proliferation and successful hepatocyte polarization. As the main epithelial cells in the liver, hepatocyte polarization, including tight junctional, cytoskeletal and intracellular trafficking components, is essential for the regenerative liver function. The tight junction, a polarized structure, forms a seal between cells, separating the basolateral and apical membrane domains when epithelial cells acquire polarity (10). Bile salt export pump (BSEP) is a member of the $\mathrm{ABC}$ superfamily of efflux transporter proteins, and is crucial in the efflux of bile salts (11). Inhibition of BSEP may lead to accumulation of cytotoxic bile salts and eventually have severe consequences such as intrahepatic cholestasis (12).

AMP-activated protein kinase (AMPK), which is a cellular metabolic sensor, has been demonstrated to be involved in liver regeneration $(13,14)$. Generally, AMPK activation switches off ATP-consuming processes and switches on ATP-generating pathways. Following partial hepatectomy $(\mathrm{PH})$, hepatocyte proliferation is associated with increased 
AMPK phosphorylation. Deletion of AMPK delays liver proliferation by affecting the G1/S transition phase (14). An additional function of AMPK activation is also evident on hepatocyte polarization (15). In collagen sandwich cultures of rat hepatocytes, AMPK regulated bile canalicular network formation and maintenance (13). AMPK activation and canalicular network formation are associated with BSEP trafficking (15).

Based on these previous studies, a hypothesis was formed that oral supplementation with FO may be able to induce AMPK activation and regulate hepatocyte polarization markers, thereby promoting the recovery of liver function during PH.

\section{Materials and methods}

Animal experiments. A total of 120 ICR mice ( $\mathrm{n}=24$ /group, $\mathrm{n}=6 /$ time interval; male; $25-30 \mathrm{~g}$; 8-15 weeks old) obtained from the Animal Experimental Center, Nanjing Drum Tower Hospital, Medical School of Nanjing University (Nanjing, China) were used for simple 70\% PH experiments. Mice were housed under conditions of $60 \%$ humidity, a temperature of $18-22^{\circ} \mathrm{C}$, a $12 \mathrm{~h}$ light/dark cycle and free access to food and water. Mice were fasted overnight prior to $\mathrm{PH}$. Anesthesia was induced with chloral hydrate $(10 \% ; 350 \mathrm{mg} / \mathrm{kg})$ by intraperitoneal (i.p.) injection. Following laparotomy, the median and left liver lobes were surgically removed resulting in $70 \% \mathrm{PH}$. Mice were sacrificed via carbon dioxide inhalation asphyxia to collect blood and liver specimens prior to $\mathrm{PH}$ (days -2 and 0 ) and following $\mathrm{PH}$ (days 1, 2, 3 and 5), and the recovery of liver mass was estimated by the liver-to-body weight ratios. The experimental design was examined and approved by the Experimental Animal Ethics Committee of the Nanjing Drum Tower Hospital, Medical School of Nanjing University (Nanjing, China).

Experimental groups. All animals were divided randomly into 5 groups ( $\mathrm{n}=24$ per group): i) Sham; ii) Control; iii) Compound C (Selleck Chemicals, Houston, TX, USA), which is the AMPK inhibitor dorsomorphin; iv) FO [comprising 40\% docosahexaenoic acid (DHA) and 40\% eicosapentaenoic acid (EPA); Wuhan Shengtianyu Biotechnology, Wuhan, China]; and v) Compound $\mathrm{C}+\mathrm{FO}$. Mice in the Sham group had laparotomy and exposure of the liver, but no PH. Mice in the Control group had 70\% PH without any treatment. Mice in the Compound $\mathrm{C}$ group had $70 \% \mathrm{PH}$ and treatment with Compound $\mathrm{C}(8 \mathrm{mg} / \mathrm{kg})$ by i.p. injection daily, beginning half an hour prior to the operation until the day prior to the indicated time. Mice in the FO group had $70 \% \mathrm{PH}$ and treatment with FO $(12 \mathrm{ml} / \mathrm{kg})$ by oral gavage daily, beginning at 2 days prior to the operation until the day prior to the indicated time. Mice in the Compound $\mathrm{C}+\mathrm{FO}$ group had $70 \% \mathrm{PH}$ and treatment with $\mathrm{FO}(12 \mathrm{ml} / \mathrm{kg})$ by oral gavage daily from 2 days prior the operation until the day prior to the indicated time and Compound C $(8 \mathrm{mg} / \mathrm{kg})$ by i.p. injection daily from half an hour prior to the operation until the day prior to the indicated time.

Western blotting. A total of $50 \mathrm{mg}$ liver tissues were lysed in cold radioimmunoprecipitation assay buffer (Beyotime Institute of Biotechnology, Haimen, China) containing 1:100 volume of phenylmethylsulfonyl fluoride. Following lysis at $4^{\circ} \mathrm{C}$ for $1 \mathrm{~h}$, cell lysates were centrifuged at $14,000 \mathrm{x}$ g for $5 \mathrm{~min}$ at $4^{\circ} \mathrm{C}$. The total protein concentration in the supernatant was determined by Bicinchoninic Acid assay (Beyotime Institute of Biotechnology). Proteins were separated by $10 \%$ SDS-PAGE $(30 \mu \mathrm{g} /$ lane) and transferred to a nitrocellulose membrane at $100 \mathrm{~V}$ for $60 \mathrm{~min}$. Following blocking in TBS $+0.1 \%$ Tween-20 (TBST) buffer containing 5\% bovine serum albumin (BSA; Absin Bioscience Inc., Shanghai, China) at $37^{\circ} \mathrm{C}$ for $1 \mathrm{~h}$, the membranes were incubated in TBST containing $2 \%$ BSA and antibodies against Occludin (cat. no. ab64482; 1:1,000; Abcam, Cambridge, UK), Claudin-3 (cat. no. sc-17662; 1:1,000; Santa Cruz Biotechnology, Inc., Dallas, TX, USA), tight junction protein ZO-1 (ZO-1; cat. no. sc-10804; 1:1,000; Santa Cruz Biotechnology, Inc.), phosphorylated (p)-AMPK (cat. no. sc-33524; 1:1,000; Santa Cruz Biotechnology, Inc.), AMPK (cat. no. sc-33524; 1:1,000; Santa Cruz Biotechnology, Inc.) and BSEP (cat. no. sc-74500; 1:1,000; Santa Cruz Biotechnology, Inc.) at $37^{\circ} \mathrm{C}$ for $1 \mathrm{~h}$. An antibody against $\beta$-actin (cat. no. 4970; 1:5,000; Cell Signaling Technology, Inc., Danvers, MA, USA) was used as an internal control. The membranes were washed three times with TBST and incubated with the following secondary antibodies at $37^{\circ} \mathrm{C}$ for $1 \mathrm{~h}$ : Goat anti-rabbit horseradish peroxidase (HRP)-conjugated antibody (cat. no. BA1055; 1:5,000; Boster Biological Technology, Pleasanton, CA, USA), HRP-goat anti-mouse antibody (cat. no. BA1051; 1:5,000; Boster Biological Technology) and HRP-rabbit anti-goat antibody (cat. no. BA1060; 1:5,000; Boster Biological Technology). Following three washes with TBST, the target proteins were detected using an Enhanced Chemiluminescence kit (WBULS0500; EMD Millipore, Billerica, MA, USA). Densitometry analysis was performed using the Intel iPP 6.0 software (Intel Corporation, Santa Clara, CA, USA).

Immunohistochemical assays. Paraffin-embedded liver tissue sections $(\sim 4 \mu \mathrm{m})$ were fixed in $4 \%$ paraformaldehyde at room temperature for $24 \mathrm{~h}$, deparaffinized and dehydrated through graded ethanol. The sections were washed 3 times with PBS for 5 min each, and then treated with $3 \%$ hydrogen peroxide for $10 \mathrm{~min}$ at room temperature to block the endogenous peroxidase activity. The sections were subsequently washed 3 times with PBS for 5 min each. Following blocking with 5\% BSA for $30 \mathrm{~min}$ at $37^{\circ} \mathrm{C}$, sections were incubated with primary antibodies against Occludin, ZO-1, BESP or Ki-67 (1:200 dilution in PBS) overnight and then with a HRP-conjugated anti-rabbit immunoglobulin $\mathrm{G}$ secondary antibody (cat. no. BA1055; 1:100; Boster Biological Technology) for $1 \mathrm{~h}$ at room temperature. Finally, the sections were incubated with 3,3-diaminobenzidine reagent for $10 \mathrm{~min}$, counterstained with hematoxylin, dehydrated and mounted for inverted phase contrast microscopy (CK30 OLYMPUS; Olympus Corporation, Tokyo, Japan) analysis.

Serum biochemical parameters. Serum expression levels of alanine aminotransferase (ALT), aspartate aminotransferase (AST), total bilirubin (TBIL), albumin (ALB) and C-reactive protein (CRP) were determined by the Laboratory of Biochemistry, Nanjing Drum Tower Hospital, Medical School of Nanjing University (Nanjing, China). 
Table I. Serum biochemical parameters following partial hepatectomy.

\begin{tabular}{|c|c|c|c|c|c|c|}
\hline Serum parameter & Day & Sham & Control & FO & Compound C & Compound $\mathrm{C}+\mathrm{FO}$ \\
\hline \multirow[t]{4}{*}{$\operatorname{ALT}(\mathrm{U} / \mathrm{l})$} & 1 & $28.4 \pm 2.2$ & $1,353.0 \pm 644.7^{\mathrm{a}}$ & $1,253.4 \pm 530.9^{a}$ & $1,910.0 \pm 499.8^{\mathrm{a}}$ & $2,198.7 \pm 390 \cdot 3^{\mathrm{a}-\mathrm{c}}$ \\
\hline & 2 & $28.0 \pm 1.7$ & $177.4 \pm 46.4^{\mathrm{a}}$ & $112.4 \pm 19.9^{\mathrm{a}, \mathrm{b}}$ & $241.5 \pm 47.8^{\mathrm{a}, \mathrm{b}}$ & $236.2 \pm 32.7^{\mathrm{a}, \mathrm{b}}$ \\
\hline & 3 & $26.6 \pm 1.2$ & $65.0 \pm 15.4^{\mathrm{a}}$ & $36.2 \pm 4.8^{b}$ & $77.9 \pm 23.9^{\mathrm{a}}$ & $78.3 \pm 24.9^{\mathrm{a}}$ \\
\hline & 5 & $27.8 \pm 1.2$ & $54.0 \pm 10.4^{\mathrm{a}}$ & $29.0 \pm 7.8^{\mathrm{b}}$ & $66.5 \pm 24.9^{\mathrm{a}}$ & $39.4 \pm 9.6^{c}$ \\
\hline \multirow[t]{4}{*}{$\operatorname{AST}(\mathrm{U} / \mathrm{l})$} & 1 & $92.8 \pm 3.4$ & $2,344.0 \pm 738.1^{\mathrm{a}}$ & $1,744.9 \pm 705.1^{\mathrm{a}, \mathrm{b}}$ & $2,597.0 \pm 316.3^{\mathrm{a}}$ & $2,186.2 \pm 243.5^{\mathrm{a}}$ \\
\hline & 2 & $90.1 \pm 2.2$ & $243.7 \pm 57.4^{\mathrm{a}}$ & $226.0 \pm 42.6^{\mathrm{a}}$ & $500.4 \pm 69.4^{\mathrm{a}, \mathrm{b}}$ & $313.3 \pm 25.8^{\mathrm{a}-\mathrm{c}}$ \\
\hline & 3 & $93.3 \pm 2.2$ & $133.3 \pm 13.6^{\mathrm{a}}$ & $134.8 \pm 30.0^{\mathrm{a}}$ & $170.1 \pm 52.1^{\mathrm{a}, \mathrm{b}}$ & $155.4 \pm 21.3^{\mathrm{a}}$ \\
\hline & 5 & $91.3 \pm 2.2$ & $120.8 \pm 38.4^{\mathrm{a}}$ & $101.2 \pm 13.3$ & $135.0 \pm 29.2^{\mathrm{a}}$ & $139.1 \pm 22.1^{\mathrm{a}}$ \\
\hline \multirow[t]{4}{*}{ TBIL $(\mu \mathrm{mol} / 1)$} & 1 & $1.1 \pm 0.4$ & $15.9 \pm 20.0$ & $4.3 \pm 1.1$ & $18.7 \pm 26.6$ & $6.4 \pm 2.5$ \\
\hline & 2 & $1.6 \pm 0.2$ & $2.7 \pm 0.2$ & $2.0 \pm 0.4$ & $6.3 \pm 2.9^{\mathrm{a}, \mathrm{b}}$ & $4.1 \pm 1.0^{\mathrm{a}, \mathrm{c}}$ \\
\hline & 3 & $1.3 \pm 0.4$ & $3.4 \pm 0.2^{\mathrm{a}}$ & $1.3 \pm 0.2^{\mathrm{b}}$ & $2.6 \pm 0.1^{\mathrm{a}, \mathrm{b}}$ & $1.6 \pm 0.3^{\mathrm{a}-\mathrm{c}}$ \\
\hline & 5 & $1.6 \pm 0.3$ & $2.0 \pm 0.2^{\mathrm{a}}$ & $0.9 \pm 0.2^{\mathrm{b}}$ & $2.0 \pm 1.7$ & $1.2 \pm 0.2$ \\
\hline \multirow[t]{4}{*}{ ALB (g/l) } & 1 & $26.9 \pm 1.5$ & $26.5 \pm 1.1$ & $26.3 \pm 1.1$ & $25.4 \pm 1.4$ & $25.6 \pm 1.0$ \\
\hline & 2 & $26.5 \pm 1.2$ & $26.5 \pm 1.1$ & $25.1 \pm 1.8$ & $25.4 \pm 1.0$ & $25.5 \pm 1.0$ \\
\hline & 3 & $27.1 \pm 1.0$ & $26.5 \pm 1.6$ & $26.6 \pm 1.5$ & $26.0 \pm 1.6$ & $25.9 \pm 1.4$ \\
\hline & 5 & $26.6 \pm 1.3$ & $26.2 \pm 1.7$ & $26.0 \pm 1.4$ & $25.5 \pm 1.4$ & $25.3 \pm 0.7$ \\
\hline \multirow[t]{4}{*}{ CRP (mg/l) } & 1 & $1.4 \pm 0.2$ & $1.5 \pm 0.1$ & $1.5 \pm 0.8$ & $1.8 \pm 0.5$ & $1.7 \pm 0.4$ \\
\hline & 2 & $1.6 \pm 0.4$ & $1.9 \pm 0.6$ & $1.4 \pm 0.8$ & $2.1 \pm 0.3$ & $2.1 \pm 0.2$ \\
\hline & 3 & $1.5 \pm 0.4$ & $2.1 \pm 0.7$ & $2.0 \pm 0.8$ & $2.3 \pm 0.4^{\mathrm{a}}$ & $1.7 \pm 0.6$ \\
\hline & 5 & $1.3 \pm 0.3$ & $2.1 \pm 0.9$ & $2.4 \pm 1.1$ & $4.9 \pm 1.8^{\mathrm{a}, \mathrm{b}}$ & $2.3 \pm 0.6^{c}$ \\
\hline
\end{tabular}

Data are presented as the mean \pm standard deviation ( $\mathrm{n}=6$ per time interval). ${ }^{\mathrm{a}} \mathrm{P}<0.05$ vs. Sham. ${ }^{\mathrm{b}} \mathrm{P}<0.05$ vs. Control. ${ }^{\mathrm{c}} \mathrm{P}<0.05$ vs. Compound $\mathrm{C}$. ALB, albumin; ALT, alanine aminotransferase; AST, aspartate aminotransferase; CRP, C-reactive protein; FO, fish oil; TBIL, total bilirubin.

Statistical analysis. GraphPad Prism software version 5.01 (GraphPad Software, Inc., La Jolla, CA, USA) and PASW statistics version 18.0 (SPSS, Inc., Chicago, IL, USA) were used for statistical analyses. Results are expressed as the mean \pm standard deviation. One-way analysis of variance followed by the Least Significant Difference post hoc test was used to detect the statistically significant variations between groups. Each experiment was performed in triplicate. $\mathrm{P}<0.05$ was considered to indicate a statistically significant difference.

\section{Results}

Perioperative oral supplementation with FO improves liver function through AMPK activation following $P H$. Serum levels of ALT, AST, TBIL, ALB and CRP were evaluated at 1, 2, 3 and 5 days following $\mathrm{PH}$ to evaluate postoperative hepatic function (Table I). Following PH, the serum levels of ALT and AST in the Control mice were significantly increased compared with Sham $(\mathrm{P}<0.05$; Table I), followed by a gradual trend to be restored to normal over time. When $\mathrm{PH}$ was accompanied by perioperative FO supplementation, the restoration of serum ALT and TBIL levels was significantly faster than those in the Control group $(\mathrm{P}<0.05$ from day 2 and day 3 post-PH, respectively; Table I). To further examine the mechanism involved in the effects of perioperative FO supplementation on liver function, AMPK was inhibited by i.p. injection of Compound C. The levels of ALT and AST were significantly higher in the Compound $\mathrm{C}$ group compared with the Control group on day 2 post-PH, with or without perioperative FO supplementation $(\mathrm{P}<0.05$; Table I). These results indicated that liver function improvement following $\mathrm{PH}$ in mice with perioperative oral FO supplementation may be mediated through AMPK signaling.

To investigate the mechanisms of FO supplementation on improving liver function through AMPK activation, hepatocyte proliferation was examined following $\mathrm{PH}$ by immunohistochemical staining for the Ki-67 proliferation marker. The proportion of Ki-67 positive hepatocytes in the livers of the FO group was significantly increased compared with the Control group on days 1,2 and 3 following $\mathrm{PH}$ $(\mathrm{P}<0.05$; Fig. 1). On day 5 following $\mathrm{PH}$, there was no difference between the FO group and the Control group (P>0.05; Fig. 1). Measurement of the liver-to-body weight ratio reflected the results of hepatocyte proliferation. Perioperative oral supplementation with FO significantly increased postoperative liver-to-body weight ratio in mice undergoing $\mathrm{PH}$ compared with Control $(\mathrm{P}<0.05$; Fig. 2). To further examine the mechanism involved in the effects of perioperative FO supplementation on hepatocyte proliferation, AMPK was inhibited by use of Compound $\mathrm{C}$ by i.p. injection. The proportion of Ki-67 positive hepatocytes was significantly decreased in the livers of the Compound C-treated group compared with the Control group from day 1 to 3 following $\mathrm{PH}$, which was not resolved by perioperative oral supplementation with FO ( $\mathrm{P}<0.05$ vs. Control; Fig. 1$)$. The discrepant results of proliferation at day 5 may be explained in that the AMPK inhibitor may have delayed the process of hepatocyte proliferation and FO treatment was unable to 


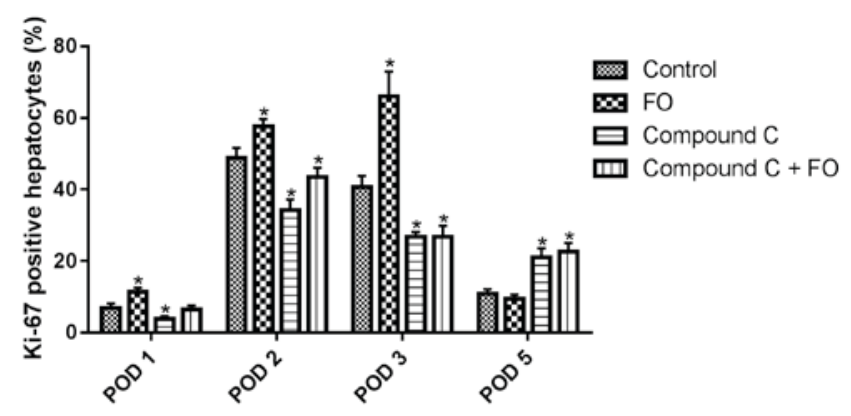

Figure 1. Effects of perioperative FO oral supplementation on hepatocyte proliferation following partial hepatectomy. Quantitation of Ki-67-positive proliferating hepatocytes in liver sections of Control, FO, Compound C and Compound $\mathrm{C}+\mathrm{FO}$ treated mice following partial hepatectomy. Data are presented as the mean \pm standard deviation; $n=6$ mice per time interval; ${ }^{*} \mathrm{P}<0.05$ vs. Control. FO, fish oil; POD, postoperative day.

reverse it, which was consistent with the liver-to-body weight ratio. The liver-to-body weight ratio was also decreased in the Compound $\mathrm{C}$ group compared with the Control group from day 1 to 5 following $\mathrm{PH}$, and treatment with perioperative $\mathrm{FO}$ supplementation did not improve it $(\mathrm{P}<0.05$; Fig. 2). These data suggested that perioperative oral supplementation with FO promoted hepatocyte proliferation in mice following $\mathrm{PH}$ and this effect was mediated through AMPK signaling.

Perioperative oral supplementation with FO promotes the expression of tight junction and BSEP proteins through AMPK activation following $\mathrm{PH}$. To further investigate the effects of perioperative FO supplementation on liver regeneration following $\mathrm{PH}$, the expression levels hepatocyte polarization markers were examined. Polarization was evaluated by western blot analysis and immunohistochemistry for the expression of epithelial differentiation markers Occludin, Claudin-3 and ZO-1, and the hepatocyte marker BSEP. Phosphorylation levels of AMPK were also evaluated as a marker of AMPK signaling activation. The ratio of $\mathrm{p}-\mathrm{AMPK} /$ total AMPK and the protein expression levels of Occludin, Claudin-3, ZO-1 and BSEP were gradually increased between day 1 and day 5 following PH in the Control group (Figs. 3 and 4). Perioperative oral supplementation with FO further enhanced this change (Figs. 3 and 4). To evaluate the effects of AMPK signaling on tight junction and BSEP protein expression, AMPK was inhibited by i.p. injection with Compound $\mathrm{C}$ (Fig. 5). The level of $\mathrm{p}$-AMPK expression was reduced in the Compound $\mathrm{C}$ group compared with the Control group $(\mathrm{P}<0.05$; Fig. $5 \mathrm{~A}$ and B), as was expression of Occludin, Claudin-3, ZO-1 and BSEP; however, the level of p-AMPK expression was still reduced in the Compound $\mathrm{C}+\mathrm{FO}$ group compared with the Control group between days 2 and $5(\mathrm{P}<0.05$; Fig. 5). In conclusion, the results demonstrated that perioperative FO oral supplementation promoted the expression of hepatocyte polarization markers in mice following $\mathrm{PH}$ and this effect was probably mediated through AMPK activation.

\section{Discussion}

Results from the present study demonstrated that oral supplementation with FO facilitated hepatocyte proliferation

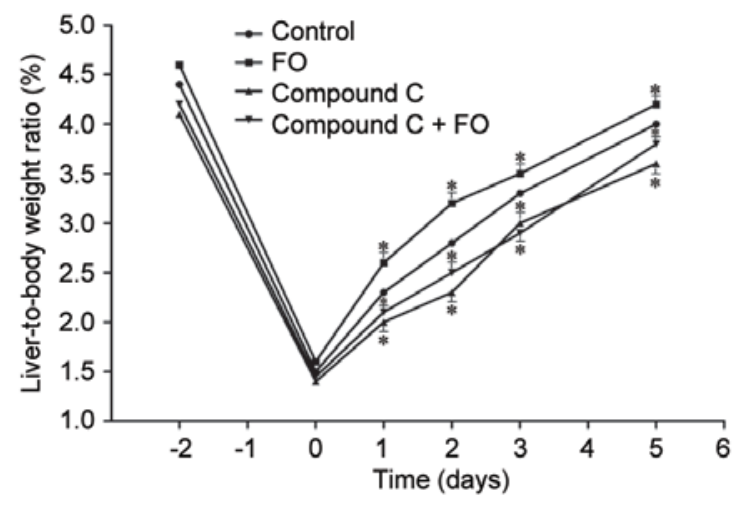

Figure 2. Effects of perioperative FO oral supplementation on liver regeneration following partial hepatectomy. Liver-to-body-weight ratios (as \% of total) for Control, FO, Compound C and Compound C + FO treated mice were measured following partial hepatectomy. Data are presented as the mean \pm standard deviation; $\mathrm{n}=6$ mice per time interval; ${ }^{*} \mathrm{P}<0.05$ vs. Control. FO, fish oil.

and the expression of hepatocyte polarization markers through AMPK activation, thereby improving liver function following PH. Serum levels of liver enzymes ALT and AST, as well as TBIL, were significantly decreased by perioperative FO oral supplementation, indicating an improvement in liver function. As hepatocyte polarization is essential for liver function, the hypothesis that FO might promote polarization of liver parenchyma to improve liver function was further tested. In addition, the role of AMPK activation in the FO-mediated effect of decreasing liver injury and improving liver function was examined. The present study revealed that AMPK activation was essential for hepatocyte proliferation and hepatocyte polarization marker expression following PH. AMPK inhibition not only suppressed cell proliferation, but also delayed cell polarization in the liver.

FO is rich in n-3 PUFA and has been demonstrated to reduce liver enzyme expression levels and to improve liver function in patients undergoing surgery (16). A meta-analysis performed by Pradelli et al demonstrated that parenteral supplementation with FO significantly reduced liver enzymes and improved liver viability in both intensive care unit (ICU) and non-ICU patients (9). Studies in humans and rodents revealed that n-3 PUFA ameliorated the degree of liver injury $(8,17)$. Previous studies from our group have also demonstrated that n-3 PUFA protected postoperative hepatic function following $70 \% \mathrm{PH}$ and prevented acute liver failure following $90 \%$ hepatectomy in rats $(18,19)$. Therefore, dietary supplementation with FO has the potential to reduce liver injury and improve liver function, which is consistent with the findings of the present study. However, the exact mechanism for this function of FO remained unclear.

The present study also demonstrated that liver function, along with expression of hepatocyte polarization markers, gradually recovered following PH. Induction of hepatocyte polarization may reduce liver injury and improve liver viability, and inhibition of hepatocyte polarization markers may delay the recovery of liver function. Hepatocytes are the main epithelial cells in the liver and they are regularly polarized (20). Hepatocyte polarization involves formation of functionally 

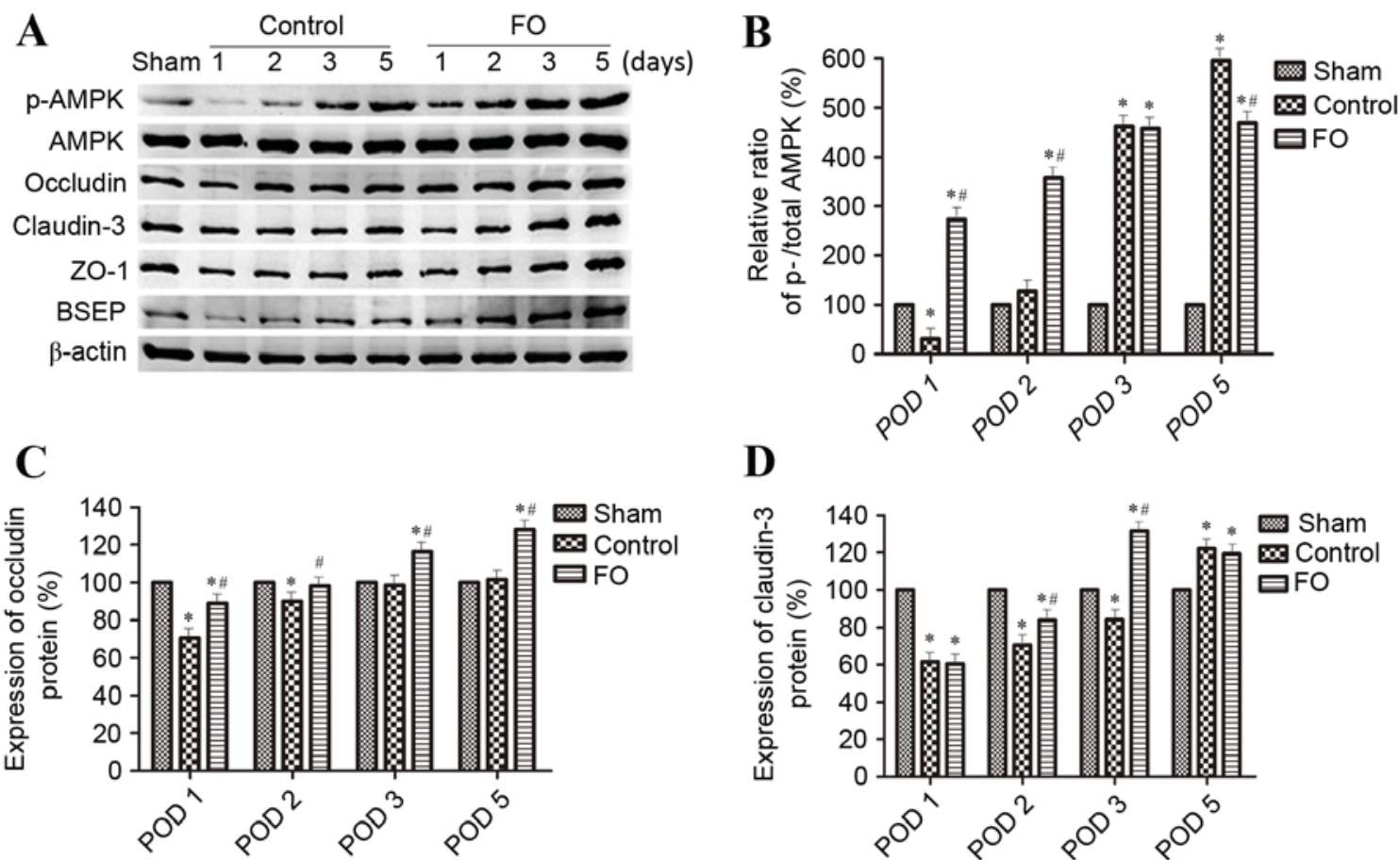

\section{D}
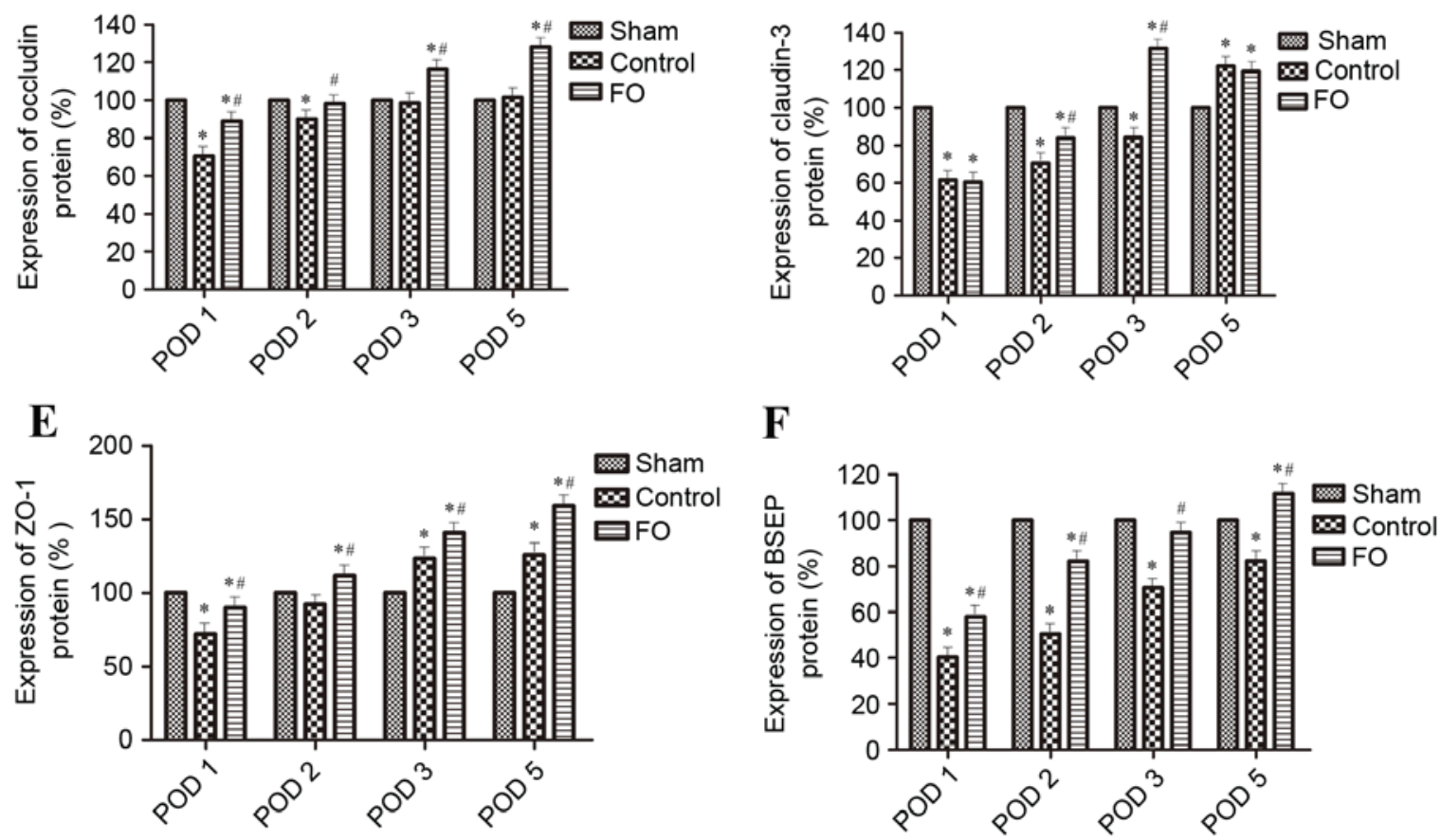

Figure 3. Effects of perioperative FO oral supplementation on hepatocyte polarization protein marker expression levels following partial hepatectomy. (A) Protein expression levels of p-AMPK, AMPK, Occludin, Claudin-3, ZO-1 and BSEP were determined by western blotting in Sham, Control and FO-treated mice on days 1, 2, 3 and 5 following partial hepatectomy; $\beta$-actin was used as an internal Control. Quantification of (B) p-AMPK:AMPK ratio, (C) Occludin, (D) Claudin-3, (E) ZO-1 and (F) BSEP levels relative to $\beta$-actin. Data are presented as the mean \pm standard deviation; ${ }^{*} \mathrm{P}<0.05$ vs. Sham; ${ }^{\#} \mathrm{P}<0.05$ vs. Control. AMPK, AMP-activated protein kinase; BSEP, bile salt export pump; FO, fish oil; p, phosphorylated; POD, postoperative day; ZO-1, tight junction protein ZO-1.

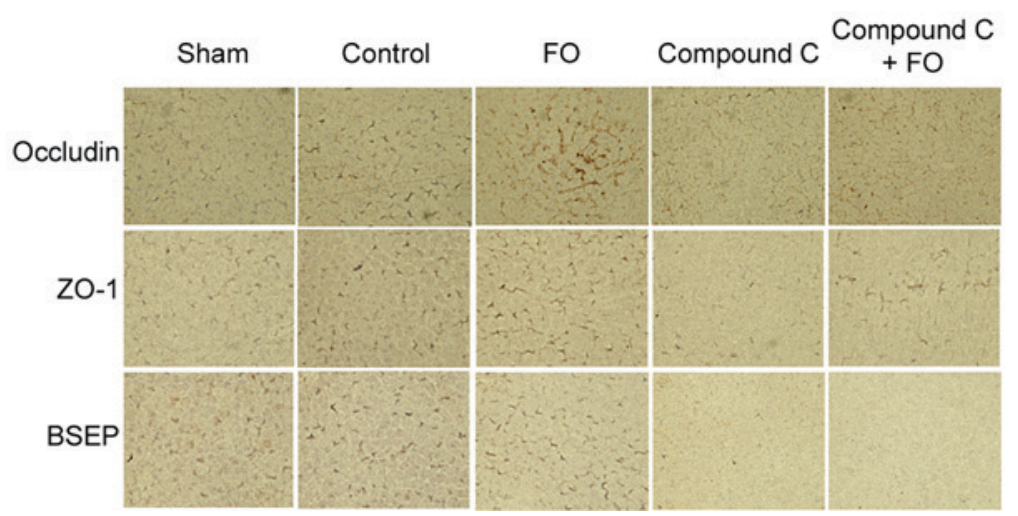

Figure 4. Expression of hepatocyte polarization marker proteins in liver tissues. Immunohistochemical staining of liver tissue sections isolated from Sham, Control, FO, Compound C and Compound C + FO treated mice at day 3 following partial hepatectomy. Liver tissue sections were stained for hepatocyte polarization markers Occludin, ZO-1 and BSEP (magnification, x20). BSEP, bile salt export pump; FO, fish oil; ZO-1, tight junction protein ZO-1.

distinct sinusoidal (basolateral) and bile canalicular (apical) plasma membrane domains that are separated by tight junction proteins, including Occludin, Claudin and ZO-1 (21). BSEP is mainly localized to the canalicular membrane of hepatocytes 


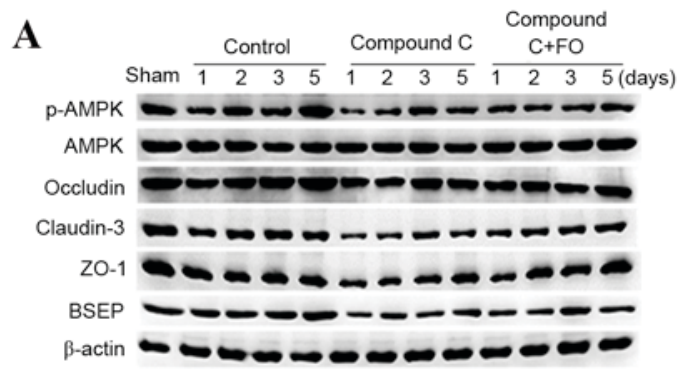

C
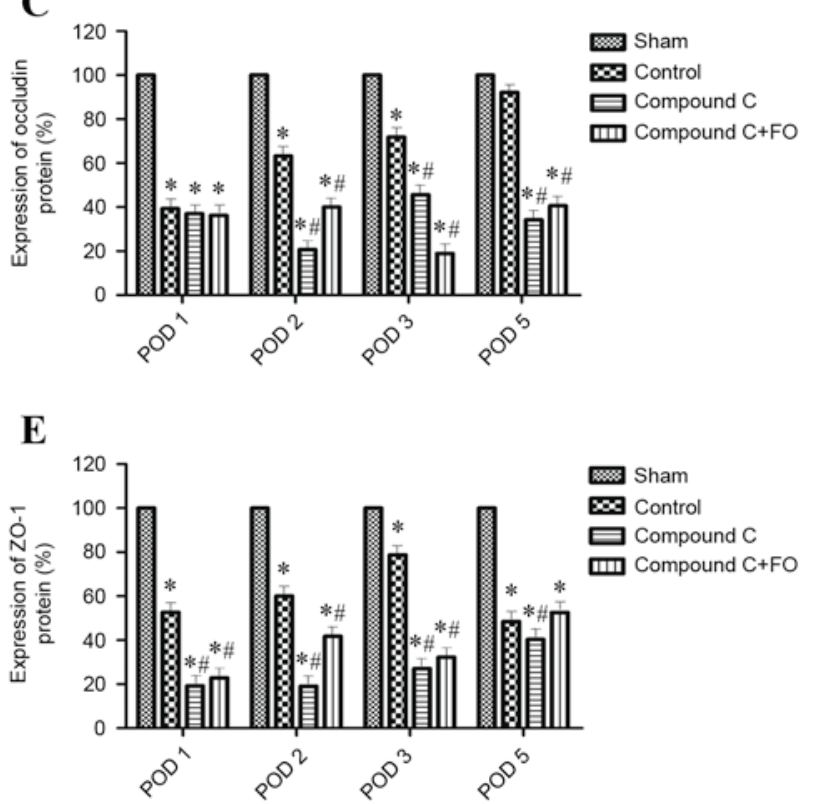

B

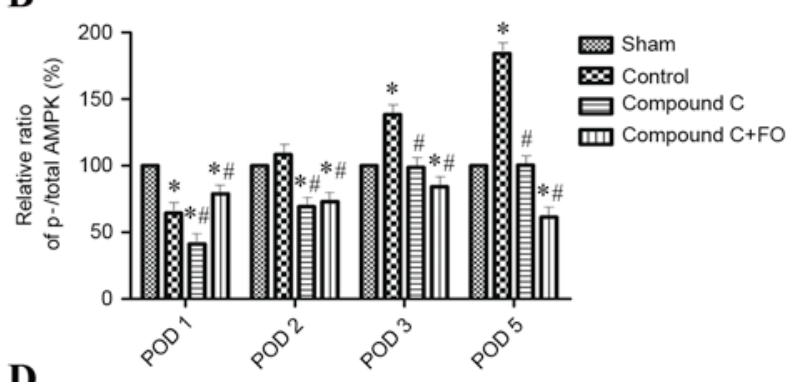

D

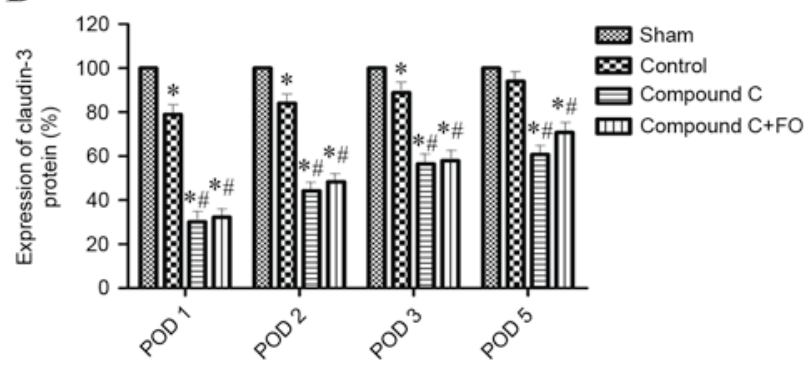

F

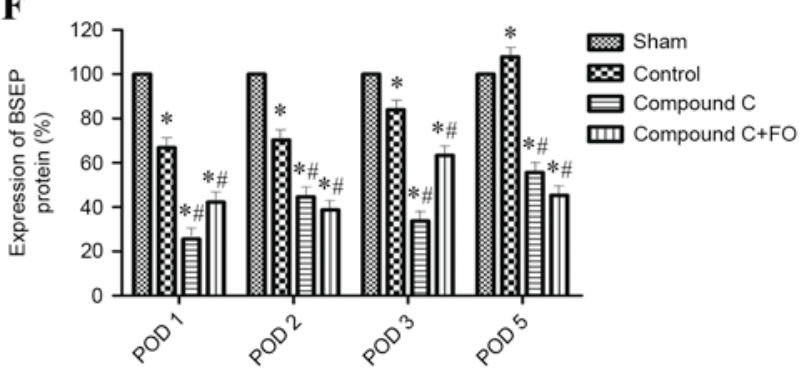

Figure 5. Effects of AMPK inhibition on expression hepatocyte polarization marker proteins following partial hepatectomy. (A) Protein expression levels of p-AMPK, AMPK, Occludin, Claudin-3, ZO-1 and BSEP were determined by western blotting in Sham, Control, Compound C and Compound C + FO mice on days 1, 2, 3 and 5 following partial hepatectomy; $\beta$-actin was used as an internal control. Quantification of (B) p-AMPK:AMPK ratio, and (C) Occludin, (D) Claudin-3, (E) ZO-1 and (F) BSEP expression levels relative to $\beta$-actin. Data are presented as the mean \pm standard deviation; ${ }^{*} \mathrm{P}<0.05$ vs. Sham; ${ }^{\#} \mathrm{P}<0.05$ vs. Control. AMPK, AMP-activated protein kinase; p, phosphorylated; BSEP, bile salt export pump; FO, fish oil; POD, postoperative day; ZO-1, tight junction protein ZO-1.

and serves a crucial role in the disposition of conjugated bile salts from the liver to the bile canaliculi (11). The inhibition of BSEP may lead to the accumulation of cytotoxic bile salts and, eventually, to severe consequences such as intrahepatic cholestasis. Cholestatic hepatocytes cause deteriorated barrier function of tight junctions (22). Therefore, hepatocyte polarization is essential for biliary secretion. Loss of polarity leads to cholestasis and liver damage (10).

In the present study, FO-mediated AMPK activation induced the expression of tight junction and BSEP proteins. AMPK is a sensor of cellular energy homeostasis that is important in energy regulation and metabolism (13). Previous studies have demonstrated that AMPK serves a role downstream of liver kinase B1 (LKB1) to confer cell polarity (23-25). In metazoan species, AMPK functions not only in controlling metabolism, but also in regulating cell structures (23). Activated LKB1 induces three major aspects of intestinal epithelial polarity in a cell-autonomous way, including forming an apical brush border, positioning junctional proteins around this brush border, and correctly sorting the basolateral and apical plasma membrane markers (24). In the polarized renal epithelial cells, AMPK also regulates tight junction assembly. Activation of AMPK facilitates tight junction assembly, whereas expression of a dominant negative AMPK construct inhibits it (25). A previous study demonstrated that hepatocyte polarization, manifested by canalicular network formation, is sequential and is associated with activation of AMPK and LKB1 in collagen sandwich cultures of rat hepatocytes (13). The canalicular network formation was accelerated by activation of AMPK and LKB1 and was blocked by inhibition of AMPK or LKB1 (13). Another previous study demonstrated that LKB1 regulates BSEP trafficking to the bile canalicular membrane, canalicular network formation and hepatocyte polarization (15).

The present study revealed that oral supplementation with FO facilitated hepatocyte polarization by AMPK activation, thereby improving liver function following $\mathrm{PH}$. However, the mechanism by which FO increases AMPK phosphorylation is not understood. Previous studies have demonstrated that AMP initiates the activation of AMPK by LKB1 $(26,27)$. In addition, it has been reported that CAMP accelerates hepatocyte polarization via the LKB1/AMPK pathway $(10,15)$. Further studies will be required to address which of these factors are 
induced by $\mathrm{FO}$ to regulate hepatocyte polarization. In addition, n-3 PUFA, including EPA and DHA, are enriched in FO. Further investigation will be required to examine in detail the relationship between n-3 PUFA and AMPK activation.

In conclusion, the present study demonstrated that oral supplementation with FO facilitated liver regeneration by AMPK activation, thereby improving liver function following $\mathrm{PH}$.

\section{Acknowledgements}

The authors acknowledge the financial support from the National Natural Science Fund of China (grant no. 81470866).

\section{References}

1. Ding BS, Cao Z, Lis R, Nolan DJ, Guo P, Simons M, Penfold ME, Shido K, Rabbany SY and Rafii S: Divergent angiocrine signals from vascular niche balance liver regeneration and fibrosis. Nature 505: 97-102, 2014.

2. Willenbring $\mathrm{H}$ and Grompe $\mathrm{M}$ : A therapy for liver failure found in the JNK yard. Cell 153: 283-284, 2013.

3. Fausto N, Campbell JS and Riehle KJ: Liver regeneration. Hepatology 43 (2 Suppl 1): S45-S53, 2006.

4. Wuestefeld T, Pesic M, Rudalska R, Dauch D, Longerich T, Kang TW, Yevsa T, Heinzmann F, Hoenicke L, Hohmeyer A, et al: A direct in vivo RNAi screen identifies MKK4 as a key regulator of liver regeneration. Cell 153: 389-401, 2013.

5. Taub R: Liver regeneration: From myth to mechanism. Nat Rev Mol Cell Biol 5: 836-847, 2004.

6. Michalopoulos GK: Principles of liver regeneration and growth homeostasis. Compr Physiol 3: 485-513, 2013.

7. He VJ: Professor Norbert Hüser: The function of immune cells for liver regeneration after partial hepatectomy. Hepatobiliary Surg Nutr 3: 52-54, 2014.

8. Svegliati-Baroni G, Candelaresi C, Saccomanno S, Ferretti G, Bachetti T, Marzioni M, De Minicis S, Nobili L, Salzano R, Omenetti A, et al: A model of insulin resistance and nonalcoholic steatohepatitis in rats: Role of peroxisome proliferator-activated receptor-alpha and n-3 polyunsaturated fatty acid treatment on liver injury. Am J Pathol 169: 846-860, 2006.

9. Pradelli L, Mayer K, Muscaritoli M and Heller AR: n-3 fatty acid-enriched parenteral nutrition regimens in elective surgical and ICU patients: A meta-analysis. Crit Care 16: R184, 2012

10. Fu D, Wakabayashi Y, Lippincott-Schwartz J and Arias IM: Bile acid stimulates hepatocyte polarization through a cAMP-Epac-MEK-LKB1-AMPK pathway. Proc Natl Acad Sci USA 108: 1403-1408, 2011

11. International Transporter Consortium; Giacomini KM, Huang SM, Tweedie DJ, Benet LZ, Brouwer KL, Chu X, Dahlin A, Evers R, Fischer V, et al: Membrane transporters in drug development. Nat Rev Drug Discov 9: 215-236, 2010.

12. Woods A, Heslegrave AJ, Muckett PJ, Levene AP, Clements M, Mobberley M, Ryder TA, Abu-Hayyeh S, Williamson C, Goldin RD, et al: LKB1 is required for hepatic bile acid transport and canalicular membrane integrity in mice. Biochem $\mathrm{J}$ 434: 49-60, 2011.
13. Fu D, Wakabayashi Y, Ido Y, Lippincott-Schwartz J and Arias IM: Regulation of bile canalicular network formation and maintenance by AMP-activated protein kinase and LKB1. J Cell Sci 123: 3294-3302, 2010.

14. Merlen G, Gentric G, Celton-Morizur S, Foretz M, Guidotti JE, Fauveau V, Leclerc J, Viollet B and Desdouets C: AMPK $\alpha 1$ controls hepatocyte proliferation independently of energy balance by regulating Cyclin A2 expression. J Hepatol 60: 152-159, 2014.

15. Homolya L, Fu D, Sengupta P, Jarnik M, Gillet JP, Vitale-Cross L, Gutkind JS, Lippincott-Schwartz J and Arias IM: LKB1/AMPK and PKA control ABCB11 trafficking and polarization in hepatocytes. PLoS One 9:e91921, 2014.

16. Stehr SN and Heller AR: Omega-3 fatty acid effects on biochemical indices following cancer surgery. Clin Chim Acta 373: 1-8, 2006.

17. Zhu XH, Wu YF, Qiu YD, Jiang CP and Ding YT: Liver-protecting effects of omega-3 fish oil lipid emulsion in liver transplantation. World J Gastroenterol 18: 6141-6147, 2012.

18. Yan XP, Wang S, Yang Y and Qiu YD: Effects of n-3 polyunsaturated fatty acids on rat livers after partial hepatectomy via LKB1-AMPK signaling pathway. Transplant Proc 43: 3604-3612, 2011.

19. Qiu YD, Wang S, Yang Y and Yan XP: Omega-3 polyunsaturated fatty acids promote liver regeneration after $90 \%$ hepatectomy in rats. World J Gastroenterol 18: 3288-3295, 2012.

20. Grosse B, Degrouard J, Jaillard D and Cassio D: Build them up and break them down: Tight junctions of cell lines expressing typical hepatocyte polarity with a varied repertoire of claudins. Tissue Barriers 1: e25210, 2013.

21. Son S, Kojima T, Decaens C, Yamaguchi H, Ito T, Imamura M, Murata M, Tanaka S, Chiba $\mathrm{H}$, Hirate $\mathrm{K}$ and Sawada N: Knockdown of tight junction protein claudin-2 prevents bile canalicular formation in WIF-B9 cells. Histochem Cell Biol 131: 411-424, 2009

22. Kojima T, Yamamoto T, Murata M, Chiba H, Kokai Y and Sawada N: Regulation of the blood-biliary barrier: Interaction between gap and tight junctions in hepatocytes. Med Electron Microsc 36: 157-164, 2003.

23. Lee JH, Koh H, Kim M, Kim Y, Lee SY, Karess RE, Lee SH, Shong M, Kim JM, Kim J and Chung J: Energy-dependent regulation of cell structure by AMP-activated protein kinase. Nature 447: 1017-1020, 2007.

24. Baas AF, Kuipers J, van der Wel NN, Batlle E, Koerten HK, Peters PJ and Clevers HC: Complete polarization of single intestinal epithelial cells upon activation of LKB1 by STRAD. Cell 116: 457-466, 2004.

25. Zhang L, Li J, Young LH and Caplan MJ: AMP-activated protein kinase regulates the assembly of epithelial tight junctions. Proc Natl Acad Sci USA 103: 17272-17277, 2006.

26. Zhang YL, Guo H, Zhang CS, Lin SY, Yin Z, Peng Y, Luo H, Shi Y, Lian G, Zhang C, et al: AMP as a low-energy charge signal autonomously initiates assembly of AXIN-AMPK-LKB1 complex for AMPK activation. Cell Metab 18: 546-555, 2013.

27. Gowans GJ, Hawley SA, Ross FA and Hardie DG: AMP is a true physiological regulator of AMP-activated protein kinase by both allosteric activation and enhancing net phosphorylation. Cell Metab 18: 556-566, 2013. 